\title{
The Quantum Instanton (QI) Model for Chemical Reaction Rates: The "Simplest" QI with One Dividing Surface ${ }^{\dagger}$
}

\author{
Charulatha Venkataraman and William H. Miller* \\ Department of Chemistry and Kenneth S. Pitzer Center for Theoretical Chemistry, University of California, and \\ Chemical Sciences Division, Lawrence Berkeley National Laboratory, Berkeley, California 94720
}

Received: September 30, 2003; In Final Form: December 10, 2003

A new version of the quantum instanton (QI) approach to thermal rate constants of chemical reactions (Miller, W. H.; Zhao, Y.; Ceotto, M.; Yang, S. J. Chem. Phys. 2003, 119, 1329) is presented, namely, the simplest QI (SQI) approximation with one dividing surface (DS), referred to here as SQI1. (The SQI approximation presented originally was applicable only with two DSs.) As with all versions of the QI approach, the rate is expressed wholly in terms of the (quantum) Boltzmann operator (which, for complex systems, can be evaluated by Monte Carlo path integral methods). Test calculations on some simple model problems show the SQI1 model to be slightly less accurate than the original version of the QI approach, but it is the easiest version to implement; it requires only a constrained free-energy calculation, location of the (transition-state) DS so as to maximize this free energy, and the curvature (second derivative) of the free energy at this maximum.

\section{Introduction}

A recent paper $^{1}$ has developed an approximate theoretical expression for the thermal rate constant of a chemical reaction that is expressed solely in terms of the quantum Boltzmann operator $e^{-\beta \hat{H}}$. Its derivation was motivated by the earlier ${ }^{2}$ semiclassical (SC) "instanton"3 approximation for rate constants and is thus referred to as the quantum instanton (QI) approximation. Applications to some standard one- and two-dimensional model problems showed it to be capable of $\pm 10-20 \%$ accuracy over a wide range of temperatures, from low temperatures deep in the tunneling regime to the high-temperature "over-barrier" limit. (More recent calculations ${ }^{4}$ have been carried out for the $\mathrm{H}+\mathrm{CH}_{4} \rightarrow \mathrm{H}_{2}+\mathrm{CH}_{3}$ reaction in full Cartesian space.) The potential usefulness of this QI approach is that the quantum Boltzmann operator can be evaluated for quite complex molecular systems by Monte Carlo (or molecular dynamics) path integration methods.5 The QI model is a type of "quantum transition-state theory", of which there are many varieties; ${ }^{6-14}$ its particular attraction is that it is not necessary to assume any reaction or tunneling path, all necessary information being contained in the Boltzmann operator.

The purpose of this paper is to develop one further aspect of the QI model that was not considered initially, namely, the variant of the model that was referred to as the simplest quantum instanton, or SQI approximation for the case that one uses a single dividing surface (DS). (The SQI given in the original work $^{1}$ is only meaningful when one uses two different DSs.) Since this is the simplest possible version of the QI idea, it is worthwhile to explore this version of the theory and see how it performs. Section 2 presents the theoretical development, section 3 presents the generalization to multidimensional systems, and section 4 shows the results of application to standard test problems. Though not quite as accurate as the original QI approximation $^{1}$ with two DSs, it is by far the easiest of all

\footnotetext{
† Part of the special issue "Fritz Schaefer Festschrift".

* Author to whom correspondence may be addressed. E-mail: miller@ cchem.berkeley.edu.
}

versions of the QI approach to implement; as summarized by eq 2.16 , it requires only a constrained free-energy calculation, location of the DS to maximize this free energy, and determination of the curvature (second derivative) of the free energy at this maximum.

\section{Theoretical Development}

We begin with the quantum instanton (QI) expression ${ }^{1}$ for the thermal rate constant $k(T)$ for the case of a single DS, here for a one-dimensional potential barrier (the multidimensional generalization will be noted below)

$k(T) Q_{\mathrm{r}}(T)=\frac{\hbar(\pi)^{1 / 2}}{\Delta H(\beta)}\left(\frac{\hbar}{2 m}\right)^{2} \cdot\left\langle x_{0}\left|e^{-\beta \hat{H} / 2}\right| x_{0}\right\rangle\left\langle\left\langle x_{0}\left|e^{-\beta \hat{H} / 2}\right| x_{0}\right\rangle\right.$

where $Q_{\mathrm{r}}$ is the reactant paritition function (per unit volume), $\Delta H(\beta)$ is an energy variance

$$
\Delta H(\beta)=\left[\frac{\left\langle x_{0}\left|e^{-\beta \hat{H} / 2} \hat{H}^{2}\right| x_{0}\right\rangle}{\left\langle x_{0}\left|e^{-\beta \hat{H} / 2}\right| x_{0}\right\rangle}-\left(\frac{\left\langle x_{0}\left|e^{-\beta \hat{H} / 2} \hat{H}\right| x_{0}\right\rangle}{\left\langle x_{0}\left|e^{-\beta \hat{H} / 2}\right| x_{0}\right\rangle}\right)^{2}\right]^{1 / 2}
$$

and $x_{0}$ is the value of $x$ for which

$$
\frac{\mathrm{d}}{\mathrm{d} x}\left\langle x\left|e^{-\beta \hat{H} / 2}\right| x\right\rangle=0
$$

The primes on the matrix elements in eq 2.1a denote differentiation with respect to the coordinate variable in the bra or ket. To develop the SQI approximation for this case of one DS, we proceed as before ${ }^{1}$ and consider the SC limit of eq 2.1; i.e., we utilize the SC approximation for the matrix elements of the Boltzmann operator ${ }^{15}$

$$
\left\langle x^{\prime}\left|e^{-\beta \hat{H} / 2}\right| x\right\rangle=\sum_{k}\left(\frac{-1}{2 \pi \hbar} \frac{\partial^{2} S_{\mathrm{k}}}{\partial x^{\prime} \partial x}\right)^{1 / 2} e^{-\mathrm{S}_{k}\left(x^{\prime}, x ; \hbar \beta / 2\right) / \hbar}
$$

where the sum in eq 2.2 is over all trajectories (in pure imaginary 


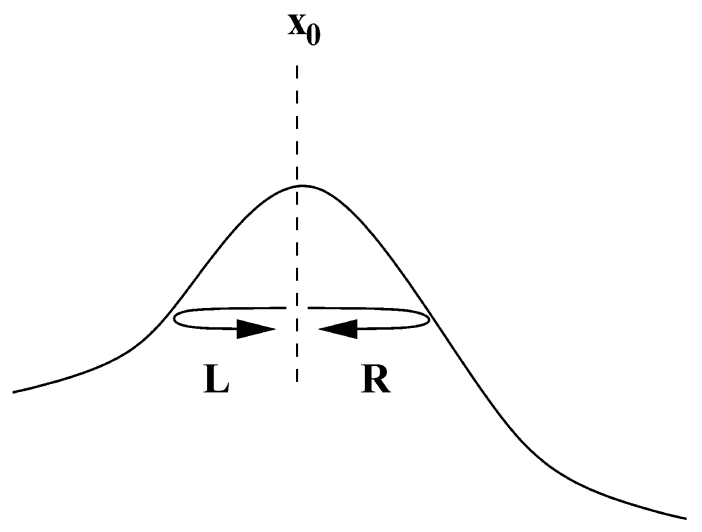

Figure 1. A sketch of a general one-dimensional potential barrier indicating the two (imaginary time) trajectories that contribute to the semiclassical approximation to the Boltzmann matrix element in eq 2.3.

time or real time on the upside-down potential surface) that go from $x$ to $x^{\prime}$ in (imaginary) time $\hbar \beta / 2$. (When using two DSs, there is only one such trajectory.) Figure 1 indicates that there will typically be two such trajectories in the present case, one that moves from $x_{0}$ to the left $(\mathrm{L})$ and back to $x_{0}$, and one that moves to the right $(\mathrm{R})$ and back, so that

$$
\begin{array}{r}
\left\langle x_{0}\left|e^{-\beta \hat{H} / 2}\right| x_{0}\right\rangle=\left(\frac{-1}{2 \pi \hbar} \frac{\partial^{2} S_{\mathrm{L}}}{\partial x^{\prime} \partial x}\right)^{1 / 2} e^{-S_{\mathrm{L}}\left(x^{\prime}, x ; \hbar \beta / 2\right) / \hbar}+ \\
\left(\frac{-1}{2 \pi \hbar} \frac{\partial^{2} S_{\mathrm{R}}}{\partial x^{\prime} \partial x}\right)^{1 / 2} e^{-S_{\mathrm{R}}\left(x^{\prime}, x ; \hbar \beta / 2\right) / \hbar}
\end{array}
$$

with $x^{\prime}=x=x_{0}$. Since

$$
\frac{\partial S_{k}\left(x^{\prime}, x\right)}{\partial x^{\prime}}=p_{k}{ }^{\prime}, \frac{\partial S_{k}\left(x^{\prime}, x\right)}{\partial x}=-p_{k}
$$

where $p_{k}\left(p_{k}{ }^{\prime}\right)$ is the initial (final) momentum for trajectory $k=$ $\mathrm{L}$ or $\mathrm{R}$, one sees that

$$
\begin{aligned}
\frac{\mathrm{d}}{\mathrm{d} x_{0}}\left\langle x_{0}\left|e^{-\beta \hat{H} / 2}\right| x_{0}\right\rangle= & \left(\frac{-1}{2 \pi \hbar} \frac{\partial^{2} S_{\mathrm{L}}}{\partial x^{\prime} \partial x}\right)^{1 / 2} e^{-S_{\mathrm{L}} / \hbar}\left(\frac{-p_{\mathrm{L}}{ }^{\prime}}{\hbar}+\frac{p_{\mathrm{L}}}{\hbar}\right)+ \\
& \left(\frac{-1}{2 \pi \hbar} \frac{\partial^{2} S_{\mathrm{R}}}{\partial x^{\prime} \partial x}\right)^{1 / 2} e^{-\mathrm{S}_{\mathrm{R}} / \hbar}\left(\frac{-p_{\mathrm{R}}{ }^{\prime}}{\hbar}+\frac{p_{\mathrm{R}}}{\hbar}\right)
\end{aligned}
$$

Thus if $x_{0}$ is chosen so that

$$
S_{\mathrm{L}}\left(x_{0}, x_{0}\right)=S_{\mathrm{R}}\left(x_{0}, x_{0}\right)
$$

then the RHS of eq 2.5 will be 0 (i.e., eq $2.1 \mathrm{c}$ will be satisfied) since (as seen in Figure 1) $p_{\mathrm{L}}{ }^{\prime}=-p_{\mathrm{L}}=-p_{\mathrm{R}}{ }^{\prime}=p_{\mathrm{R}}$. For a symmetric barrier, it is clear that $x_{0}$ is at the maximum of the potential $V(x)$, the conventional transition state.

Utilizing eqs $2.3-2.5$, one thus has the following relations in the SC limit

$$
\begin{gathered}
\left\langle x_{0}\left|e^{-\beta \hat{H} / 2}\right| x_{0}\right\rangle=2 e^{-\mathrm{S} / \hbar}\left(\frac{-1}{2 \pi \hbar} \frac{\partial^{2} S}{\partial x^{\prime} \partial x}\right)^{1 / 2} \\
\left\langle x_{0}\left|e^{-\beta \hat{H} / 2}\right| x_{0}\right\rangle=2 e^{-\mathrm{S} / \hbar}\left(\frac{-1}{2 \pi \hbar} \frac{\partial^{2} S}{\partial x^{\prime} \partial x}\right)^{1 / 2}\left(\frac{p_{0}^{2}}{\hbar^{2}}-\frac{1}{\hbar} \frac{\partial^{2} S}{\partial x^{\prime} \partial x}\right)
\end{gathered}
$$

where $S=S_{\mathrm{L}}\left(x_{0}, x_{0}\right)=S_{\mathrm{R}}\left(x_{0}, x_{0}\right)$ and $p_{0}=p_{\mathrm{L}}{ }^{\prime}=-p_{\mathrm{L}}=-p_{\mathrm{R}}{ }^{\prime}=$ $p_{\mathrm{R}}$. For future reference we also note that

$$
\begin{aligned}
“\left\langle x_{0}\left|e^{-\beta \hat{H} / 2}\right| x_{0}\right\rangle= & \left\langle x_{0}\left|e^{-\beta \hat{H} / 2}\right| x_{0}\right\rangle "= \\
& 2 e^{-S / \hbar}\left(\frac{-1}{2 \pi \hbar} \frac{\partial^{2} S}{\partial x^{\prime} \partial x}\right)^{1 / 2}\left(\frac{p_{0}{ }^{2}}{\hbar^{2}}-\frac{1}{\hbar} \frac{\partial^{2} S}{\partial x^{\prime 2}}\right)
\end{aligned}
$$

Using eqs $2.7 \mathrm{a}$ and $2.7 \mathrm{~b}$ in eq 2.1a thus gives the SC limit of the QI rate constant as

$$
\begin{array}{r}
\left.k_{\mathrm{SC}-\mathrm{QI}} Q_{\mathrm{r}}=\frac{\hbar(\pi)^{1 / 2}}{\Delta H}\left(\frac{\hbar}{2 m}\right)^{2} 4 e^{-2 \mathrm{~S} / \hbar(} \frac{-1}{2 \pi \hbar} \frac{\partial^{2} S}{\partial x^{\prime} \partial x}\right)\left(\frac{p_{0}{ }^{2}}{\hbar^{2}}-\right. \\
\left.\frac{1}{\hbar} \frac{\partial^{2} S}{\partial x^{\prime} \partial x}\right)
\end{array}
$$

As before, we now equate this to the original SC instanton rate expression $^{2}$

$$
k_{\mathrm{SC}-\mathrm{I}} Q_{\mathrm{r}}=\frac{\Delta H}{2 \hbar(\pi)^{1 / 2}} e^{-2 \mathrm{~S} / \hbar}
$$

which implies that

$$
\Delta H^{2}=\left(\frac{\hbar}{m}\right)^{2}\left[\left(\frac{\partial^{2} S}{\partial x^{\prime} \partial x}\right)^{2}-\frac{p_{0}{ }^{2}}{\hbar} \frac{\partial^{2} S}{\partial x^{\prime} \partial x}\right]
$$

solving this equation for $\partial^{2} S / \partial x^{\prime} \partial x$ gives

$$
\frac{-\partial^{2} S}{\partial x^{\prime} \partial x}=\frac{1}{2}\left(\frac{-p_{0}^{2}}{\hbar}+\sqrt{\frac{+p_{0}^{4}}{\hbar^{2}}+\frac{2 m \Delta H^{2}}{\hbar^{2}}}\right)
$$

Finally, substituting this result, eq $2.10 \mathrm{~b}$, back into eq 2.8 (and using eq 2.7a) gives the simplest quantum instanton for $1 \mathrm{DS}$ (SQI1) as

$k_{\mathrm{SQI} 1} Q_{\mathrm{r}}=\frac{(\pi)^{1 / 2}}{2} \frac{\hbar}{2 m}\left(\left\langle x_{0}\left|e^{-\beta \hat{H} / 2}\right| x_{0}\right\rangle\right)^{2}\left(z+\left(1+z^{2}\right)^{1 / 2}\right)$

where $z$ is the dimensionless variable

$$
z=\frac{1}{\Delta H} \frac{p_{0}^{2}}{2 m}
$$

To complete the SQI1 model, one needs a way to determine the momentum $p_{0}$, or more specifically, $p_{0}^{2} / 2 m$, the kinetic energy at the DS (i.e., at the transition state). A simple prescription for doing so is based on classical conservation of energy (for the imaginary time trajectory)

$$
E(\beta)=-\frac{p_{0}^{2}}{2 m}+V\left(x_{0}\right) \text { or } \quad \frac{p_{0}^{2}}{2 m}=V\left(x_{0}\right)-E(\beta)
$$

where $E(\beta)$ is given by

$$
E(\beta)=\frac{\left\langle x_{0}\left|e^{-\beta \hat{H} / 2} \hat{H}\right| x_{0}\right\rangle}{\left\langle x_{0}\left|e^{-\beta \hat{H} / 2}\right| x_{0}\right\rangle}
$$

We have indeed used eq 2.12 in eq $2.11 \mathrm{~b}$, and the rate constants obtained are reasonably good. We do not consider this a satisfactory approach, however, because it does not generalize to the multidimensional case; eq 2.12 gives the total kinetic energy on the DS, while it is clear (e.g., by considering the separable limit) it should be only the kinetic energy in the reaction coordinate. A definition of $p_{0}^{2} / 2 m$ that does generalize 
correctly is obtained by averaging eqs $2.7 \mathrm{~b}$ and $2.7 \mathrm{c}$, which gives

$$
\begin{array}{r}
\frac{p_{0}^{2}}{2 m}=\frac{\hbar^{2}}{4 m}\left(\frac{\left\langle x_{0}\left|e^{-\beta \hat{\mathrm{H}} / 2}\right| x_{0}\right\rangle+{ }^{\prime} \cdot\left\langle x_{0}\left|e^{-\beta \hat{H} / 2}\right| x_{0}\right\rangle}{\left\langle x_{0}\left|e^{-\beta \hat{H} / 2}\right| x_{0}\right\rangle}\right)+ \\
\frac{\hbar}{4 m}\left(\frac{\partial^{2} S}{\partial x^{\prime} \partial x}+\frac{\partial^{2} S}{\partial x^{\prime 2}}\right)
\end{array}
$$

In the high-temperature limit, $S\left(x^{\prime}, x\right) \propto\left(x^{\prime}-x\right)^{2}$ so that the last term in eq 2.13 vanishes, and it is negligible compared to the first term in the low-temperature limit. To complete the SQI1 model we thus choose

$$
\frac{p_{0}{ }^{2}}{2 m}=\frac{\hbar^{2}}{4 m}\left(\frac{\left\langle x_{0}\left|e^{-\beta \hat{H} / 2}\right| x_{0}\right\rangle \cdot+"\left\langle x_{0}\left|e^{-\beta \hat{H} / 2}\right| x_{0}\right\rangle}{\left\langle x_{0}\left|e^{-\beta \hat{H} / 2}\right| x_{0}\right\rangle}\right)
$$

Perhaps the most convenient aspect of eq 2.14 is that it can also be expressed as

$$
\frac{p_{0}^{2}}{2 m}=\frac{\hbar^{2}}{8 m} \frac{\mathrm{d}^{2}}{\mathrm{~d} x_{0}^{2}} \ln \left[\left\langle x_{0}\left|e^{-\beta \hat{H} / 2}\right| x_{0}\right\rangle\right]
$$

where, as eq 2.1c implies, $x_{0}$ is determined by

$$
\frac{\mathrm{d}}{\mathrm{d} x_{0}} \ln \left[\left\langle x_{0}\left|e^{-\beta \hat{H} / 2}\right| x_{0}\right\rangle\right]=0
$$

i.e., one varies $x_{0}$ to find the value for which $\ln \left[\left\langle x_{0}\left|e^{-\beta \hat{H} / 2}\right| x_{0}\right\rangle\right]$ is a minimum, and $p_{0}^{2} / 2 m$ of eq $2.15 \mathrm{a}$ is given in terms of the curvature at this minimum.

To summarize, one can also write the final result in thermodynamic language. Thus if the free energy $G\left(x_{0}\right)$ as a function of the reaction coordinate is defined by

$$
\beta G\left(x_{0}\right) \equiv-\ln \left[\left\langle x_{0}\left|e^{-\beta \hat{H} / 2}\right| x_{0}\right\rangle\right]^{2}
$$

then $x_{0}$ is determined by the maximum of this free energy

$$
G^{\prime}\left(x_{0}\right)=0
$$

and the dimensionless variable $z$ (of eq 2.11b) given in terms of its curvature at the maximum

$$
z=\frac{-\hbar^{2}}{16 m} \frac{\beta G^{\prime \prime}\left(x_{0}\right)}{\Delta H(\beta)}
$$

The SQI1 expression for the rate constant is then

$$
k_{\mathrm{SQI} 1} Q_{\mathrm{r}}=\frac{(\pi)^{1 / 2}}{2} \frac{\hbar}{2 m} e^{-\beta \mathrm{G}\left(x_{0}\right)}\left(z+\left(1+z^{2}\right)^{1 / 2}\right)
$$

\section{Multidimensional Generalization}

In light of the previous QI paper, ${ }^{1}$ the multidimensional generalization of the SQI1 rate constant is straightforward. For simplicity, we consider the case that the coordinates of the system are $(x, \boldsymbol{Q})$ where $x$ is the reaction coordinate. In terms of the thermodynamic language of eqs 2.16 , the multidimensional expression for the free energy is

$$
\beta G\left(x_{0}\right)=-\ln \left[\int \mathrm{d} \boldsymbol{Q}_{\mathbf{1}} \int \mathrm{d} \boldsymbol{Q}_{\mathbf{2}}\left(\left\langle x_{0} \boldsymbol{Q}_{\mathbf{2}}\left|e^{-\beta \hat{H} / 2}\right| x_{0} \boldsymbol{Q}_{\mathbf{1}}\right\rangle\right)^{2}\right]
$$

and then eqs $2.16 \mathrm{~b}-\mathrm{d}$ pertain as written. The multidimensional energy variance $\Delta H$ is as given before

$$
\begin{aligned}
& \Delta H(\beta)^{2}= \\
& \frac{\int \mathrm{d} \boldsymbol{Q}_{1} \int \mathrm{d} \boldsymbol{Q}_{2}\left[\left\langle x_{0} \boldsymbol{Q}_{2}\left|e^{-\beta \hat{H} / 2} \hat{H}^{2}\right| x_{0} \boldsymbol{Q}_{1}\right\rangle\left\langle x_{0} \boldsymbol{Q}_{2}\left|e^{-\beta \hat{H} / 2}\right| x_{0} \boldsymbol{Q}_{1}\right\rangle-\left(\left\langle x_{0} \boldsymbol{Q}_{2}\left|e^{-\beta \hat{H} / 2} \hat{H}\right| x_{0} \boldsymbol{Q}_{1}\right\rangle\right)^{2}\right]}{\int \mathrm{d} \boldsymbol{Q}_{\mathbf{1}} \int \mathrm{d} \boldsymbol{Q}_{\mathbf{2}}\left(\left\langle x_{0} \boldsymbol{Q}_{2}\left|e^{-\beta \hat{H} / 2}\right| x_{0} \boldsymbol{Q}_{1}\right\rangle\right)^{2}}
\end{aligned}
$$

The generalization of eqs 3.1 and 3.2 to the case of a more general form of the DS, e.g., specified by some function (the generalized reaction coordinate) $s(\boldsymbol{q})=0$, where $\boldsymbol{q}=(x, \boldsymbol{Q})$ denotes all the (Cartesian) coordinates of the system, has been given (and implemented) in ref 4.

\section{Test Calculations}

The first example we consider is the standard one-dimensional symmetric Eckart potential barrier

$$
V(x)=V_{0} \operatorname{sech}^{2}(a x)
$$

with parameters $V_{0}=0.425 \mathrm{eV}, a=1.36 \mathrm{au}$, and $m=1060$ $\mathrm{au}$, which correspond approximately to the $\mathrm{H}+\mathrm{H}_{2}$ reaction. For these elementary one-dimensional examples, it is simplest to evaluate the necessary matrix elements of the Boltzmann operator by quantum basis-set methods (we used a discrete variable representation), ${ }^{16}$ though the practical interest in the quantum instanton approach is that the Boltzman operator can be evaluated for very complex systems by Monte Carlo path integral methods. ${ }^{5}$

Figure $2 \mathrm{a}$ first displays an Arrhenius plot of the rate constant given by the SQI1 model, i.e., eq 2.11 with 2.14 over the range $T=100-2000 \mathrm{~K}$, along with the exact quantum rate, showing that on this scale the model gives excellent results. (For this symmetric case, the location of the transition state DS $x_{0}$ is always $x_{0}=0$.) To show the accuracy of the model more precisely, Figure $2 \mathrm{~b}$ plots the ratio of the SQI1 rate to the exact quantum rate as a function of $1 / T$; it is $\sim 10-15 \%$ too small at the lowest and highest temperatures, with the greatest deviation (a factor of 1.4 ) at intermediate temperature. By comparison of these results to the earlier QI model with one DS, the present SQI1 model is better at the lowest and highest temperature but not as accurate in the intermediate temperature regime.

The second example is the asymmetric version of the Eckart potential

$$
V(x)=\frac{V_{0}(1-\alpha)}{1+e^{-2 a x}}+\frac{V_{0}\left(1+(\alpha)^{1 / 2}\right)^{2}}{4 \cosh ^{2}(a x)}
$$

with parameters $V_{0}=0.425 \mathrm{eV}, a=1.36 \mathrm{au}, \alpha=1.25$, and $m$ $=1060 \mathrm{au}$; the barrier heights are 0.425 and $0.531 \mathrm{eV}$ from the left and right sides of the barrier, respectively. As above, we first show in Figure 3a, an Arrhenius plot of the SQI1 rate constant, for the range $T=150-2000 \mathrm{~K}$, again displaying very good agreement with the exact quantum rate over this large range of temperature. Here the location of the DS, $x_{0}$, as determined by eq $2.1 \mathrm{c}$ or $2.15 \mathrm{~b}$, varies with temperature. Figure $3 b$ shows the ratio of the SQI1 rate to the exact quantum value over this range of temperature. This plot is very similar to the symmetric case in Figure $2 b$ except at the lowest temperatures $(<200 \mathrm{~K})$ where the SQI1 rate becomes increasingly too large (off by a factor of $\sim 1.4$ at $150 \mathrm{~K}$ ). Comparing these results to those given previously by the one DS version of the QI model, 

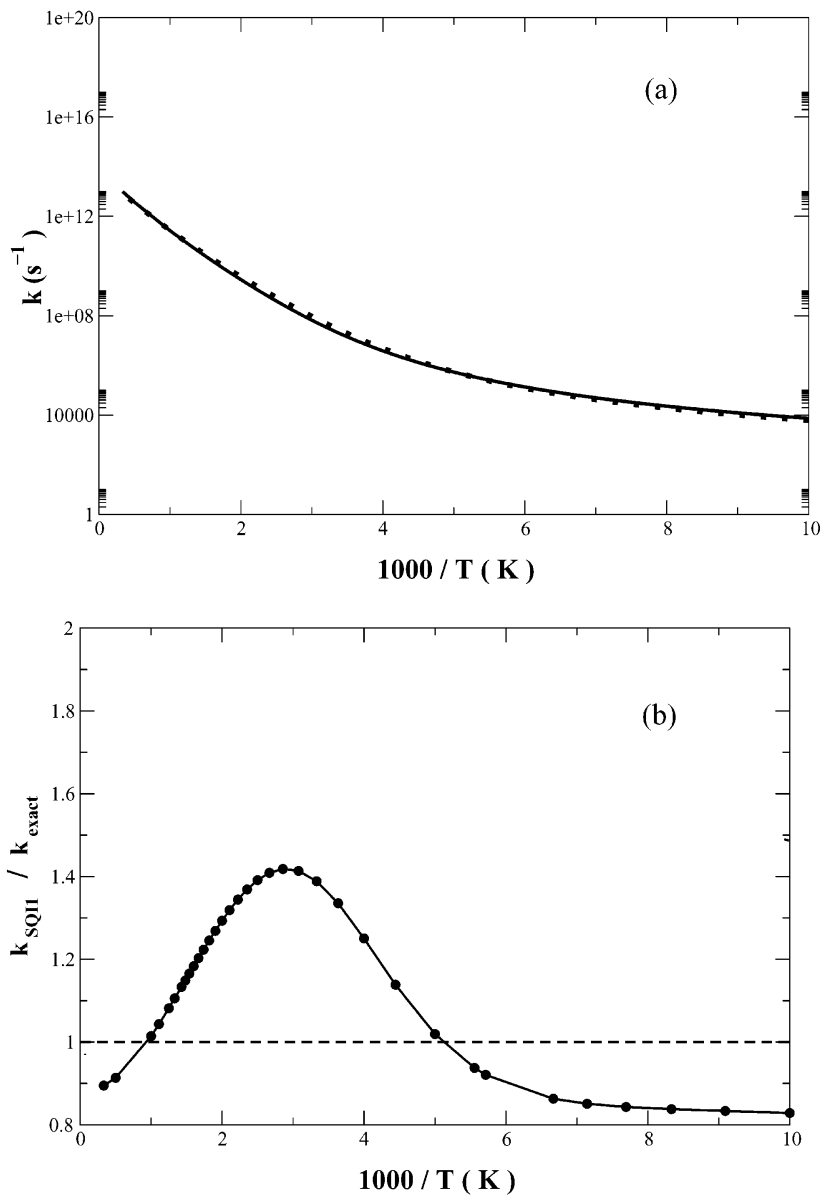

Figure 2. (a) Arrhenius plot of the rate constant given by the SQI1 model for the symmetric Eckart potential of eq 4.1. The solid curve is the exact result, and the dotted curve is the SQI1 result. (b) Ratio of the SQI1 rate constant to the exact quantum value for the symmetric Eckart potential.

the deviations of both from the correct quantum values are qualitatively similar, but those of the present SQI1 model are larger.

Finally, though it is beyond the scope of this paper, we note that the deviations of the SQI1 rates from the correct quantum values (Figures $2 \mathrm{~b}$ and $3 \mathrm{~b}$ ) are similar if plotted as a function of the dimensionless variable $z$ of eq 2.11b (with 2.14). The largest deviation, a factor of $\sim 1.4-1.5$, occurs at $z \sim 1$. It is thus possible to introduce an empirical correction factor (i.e., a function of $z$ ) that significantly corrects this generic behavior of the SQI1 model. For example, if the factor $(\pi)^{1 / 2} / 2(z+(1$ $\left.\left.+z^{2}\right)^{1 / 2}\right)$ in eq $2.11 \mathrm{a}$ and $2.16 \mathrm{~d}$ is replaced by $5 z / 4+\left((z-1)^{2}\right.$ $\left.+(1 / 4)^{2}\right)^{1 / 2}$, then the SQI1 rate agrees with the exact quantum value to within a few percent for all temperature in Figure 2 and down to $\sim 250 \mathrm{~K}$ in Figure 3 . It remains to be seen, of course, how universal this (or any) empirical correction factor may be.

\section{Concluding Remarks}

The analysis presented in section 2 shows that it is indeed possible to derive a version of the simplest quantum instanton model that utilizes a single DS, designated here SQI1. The rate expression is given by eq 2.11 with 2.14 , or equivalently by eq 2.16 in thermodynamic language and its multidimensional generalization by eqs 3.1 and 3.2 .

Though not as accurate for these test problems as the original QI model with two DSs, the SQI1 model is still of useful
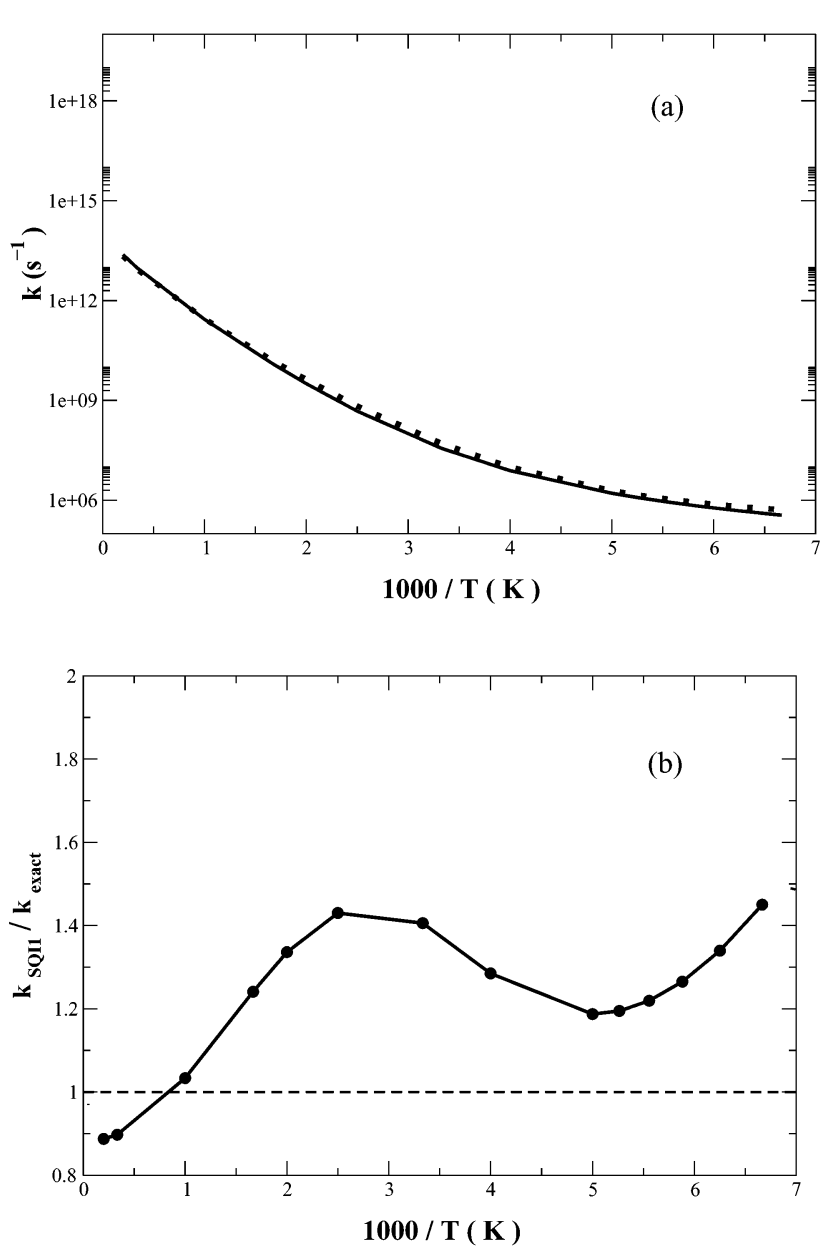

Figure 3. (a) Arrhenius plot of the rate constant given by the SQI1 model for the asymmetric Eckart potential of eq 4.2. The solid curve is the exact result, and the dotted curve is the SQI1 result. (b) Ratio of the SQI1 rate constant to the exact quantum value for the asymmetric Eckart potential.

accuracy (with empirical corrections being possible to make it even more accurate), and it is much easier to implement. For example, eqs 2.16 show that it is necessary only to carry out a rather standard constrained free-energy calculation, choose the transition state DS by (an again fairly standard) maximum freeenergy criterion, and then the rate is given by this maximum free energy and a function of the dimensionless parameter $z$, which is expressed in terms of the curvature of the free energy at its maximum.

Acknowledgment. This work has been supported by the Director, Office of Science, Office of Basic Energy Sciences, Chemical Sciences Division of the U. S. Department of Energy under Contract No. DE-AC03-76SF00098 and by National Science Foundation Grant CHE-0096576.

\section{References and Notes}

(1) Miller, W. H.; Zhao, Y.; Ceotto, M.; Yang, S. J. Chem. Phys. 2003, $119,1329$.

(2) Miller, W. H. J. Chem. Phys. 1975, 62, 1899.

(3) The term "instanton" for these types of imaginary time semiclassical approximations was introduced by others; cf. Coleman, S. Phys. Rev. D 1977, 15, 2929.

(4) Zhao, Y.; Yamamoto, T.; Miller, W. H. Path Integral Calculation of Thermal Rate Constants within the Quantum Instanton Approximation: Application to the $\mathrm{H}+\mathrm{CH}_{4} \rightarrow \mathrm{H}_{2}+\mathrm{CH}_{3}$ Hydrogen Abstraction Reaction in Full Cartesian Space. J. Chem. Phys. In press.

(5) (a) Classical and Quantum Dynamics in Condensed Phase Simulations; Berne, B. J., Ciccotti, G., Coker, D. F., Eds.; World Scientific: Singapore, 1998. (b) Ceperley, D. M. Rev. Mod. Phys. 1995, 67, 279. (c) 
Doll, J. D.; Freeman, D. L. Adv. Chem. Phys. 1989, 73, 289. (d) Doll, J. D.; Freeman, D. L.; Beck, T. L. Adv. Chem. Phys. 1994, 78, 61.

(6) Miller, W. H. J. Chem. Phys. 1974, 61, 1823.

(7) Truhlar, D. G. In The Theory of Chemical Reaction Dynamics; Baer,

M., Ed.; Chemical Rubber: Boca Raton, 1985; Vol. 4, Chapter 2.

(8) Tromp, J. W.; Miller, W. H. J. Phys. Chem. 1986, 90, 3482.

(9) (a) Voth, G. A.; Chandler, D.; Miller, W. H. J. Chem. Phys. 1989

91, 7749. (b) Jang, S.; Voth, G. A. J. Chem. Phys. 2000, 112, 8747. (c)

Geva, E.; Shi, Q.; Voth, G. A. J. Chem. Phys. 2001, 115, 9209.
(10) Voth, G. A. Chem. Phys. Lett. 1990, 170, 289.

(11) Truhlar, D. G.; Garrett, B. C. J. Phys. Chem. 1992, 96, 6515.

(12) Hansen, N. F.; Andersen, H. C. J. Chem. Phys. 1994, 101 6032.

(13) Hansen, N. F.; Andersen, H. C. J. Phys. Chem. 1996, 100, 1137.

(14) Pollak, E.; Liao, J. L. J. Chem. Phys. 1998, 108, 2733.

(15) Miller, W. H. J. Chem. Phys. 1971, 55, 3146.

(16) Colbert, D. T.; Miller, W. H. J. Chem. Phys. 1992, 96, 1982. 